\title{
Preferencias relacionales de género en el contexto escolar: Una nueva medida para el diagnóstico de relaciones de género en educación
}

\author{
Gender Relationship Preferences in the School Contexts: A new measure for assessment of \\ gender relationships in education
}

\author{
García-Pérez, Rafael (1); Ruiz-Pinto, Estrella ${ }^{(2)}$; Rebollo-Catalán, Ángeles ${ }^{(1)}$ \\ (1) Universidad de Sevilla (2) Universidad de Sevilla
}

\begin{abstract}
Resumen
Este estudio evalúa un procedimiento nuevo para el análisis “micro-sociométrico" de los patrones relacionales de género en el alumnado adolescente, lo que se analiza aplicando Análisis de Correspondencias Múltiples (ACM). El propósito del estudio es desarrollar y validar la Escala de Preferencias Relacionales de Género (EPRG), con el fin de obtener datos válidos y fiables que permitan reconocer la predisposición de las chicas y los chicos adolescentes a relacionarse con sus iguales; y, si las relaciones que establecen están condicionadas por la socialización diferencial de género que propone el patriarcado (Bosch, Ferrer \& Alzamora, 2006; Lagarde, 1996; Lomas, 2007; Lorente, 2007; Rodríguez-Mosquera, 2011; Simón, 2010; Tomé \& Tonucci, 2013). La muestra está compuesta por 6.000 estudiantes adolescentes, de los cuales el 50,8\% son mujeres y el 49,2\% son hombres, con una media de edad de 13,82 años. Se demuestra validez de constructo y se obtiene una alta fiabilidad (Alpha de Cronbrach = .92) en la determinación de las asignaciones de roles y estereotipos de género en el aula escolar, considerando al tiempo las “distancias sociales” y la estructura electiva de chicos y chicas en las aulas. La obtención con ACM de mapas "micro-sociométricos” facilita la interpretación de resultados, coincidentes con otras aproximaciones teóricas, identificando las “distancias sociales” entre personas, categorías y géneros. Los resultados destacan actividades relacionales que muestran patrones de género muy marcados tales como compartir la ropa o elegir pareja, así como las conductas relacionales asociadas a la diferenciación inequívoca inter-intra géneros.
\end{abstract}

\section{Palabras clave:}

Coeducación; Adolescencia; Relaciones entre Iguales; Diferencias de Género, Análisis Sociométrico; Diagnóstico en Educación; Fiabilidad y Validez; Distancia Social; Escalas; Análisis de Correspondencias Múltiples (ACM).

\begin{abstract}
This study evaluates a new methodological procedure for "micro-sociometric" analysis of gender relationship patterns in the adolescent students, which is analyzed using Multiple Correspondence Analysis (MCA). The purpose of the study is to develop and assessment of the Gender Relationship Preferences Scale (GRPS), in order to obtain valid and reliable data to recognize the willingness of the young (girls and boys) to interact with their peers; and to determine whether the relationships established between them are conditioned by the gender differential socialization proposed by patriarchy (Bosch, Ferrer \& Alzamora, 2006; Lagarde, 1996; Lomas, 2007; Lorente, 2007; Rodríguez-Mosquera, 2011; Simón, 2010; Tomé \& Tonucci, 2013). The sample size is 6000 cases, adolescent students, of whom $50.8 \%$ are women and $49.2 \%$ are men, with a mean age of 13.82 years. The construct validity is tested and it has obtained a high reliability (Cronbach's alpha $=.92$ ) for the determining the allocation of roles and gender stereotypes in the classroom, what it is obtained while considering the "social distance" and elective structure of boys and girls in classrooms. Maps "micro-sociometric", obtained with MCA, facilitates the interpretation of the results, which are consistent with other
\end{abstract}

Fecha de recepción 04 Septiembre 2015

\section{Fecha de} aprobación 22 Enero 2016

Fecha de publicación 23 Febrero 2016 


\begin{abstract}
theoretical approaches, identifying the "social distance" between people, categories and both genders. The results highlight relational activities showing very marked gender patterns such as sharing clothing or choosing a couple, and the relational behaviors associated with inter-intra gender differentiation.
\end{abstract}

\title{
Keywords:
}

Coeducation; Adolescence; Peer Relations; Gender Differences; Sociometric Analysis; Educational Assessment; Reliability \& Validity; Social Distance; Scales; Multiple Correspondence Analysis (MCA).

Este artículo presenta un estudio sobre patrones relacionales de género en el contexto escolar. Aunque el tema de las diferencias de género se ha abordado, aun recientemente, con una lógica causal comparativa como factor de diferenciación (Murillo \& Hernández-Castilla, 2015) y también estudiando exclusivamente a mujeres (Jiménez-Cortés, Rebollo-Catalán, García-Pérez \& Buzón-García, 2015), en este caso, el enfoque que adoptamos es relacional, aportando una novedosa perspectiva dentro de la aproximación sociométrica en las escuelas y se aborda considerando una amplia muestra de alumnado escolar de ambos géneros. Se ha diseñado $\mathrm{y}$ validado un procedimiento diagnóstico original, denominado "Escala de Preferencias Relacionales de Género” (EPRG), que nos sirve para desarrollar una nueva métrica socioeducativa. A través de este procedimiento se reconoce la predisposición de chicas y chicos adolescentes para relacionarse con sus iguales (inter-intra géneros), así como las consecuencias de su socialización diferencial de género que se observan a través de un diverso conjunto de actividades relacionales en las que expresan sus preferencias.

El reconocimiento de este tipo de patrones relacionales de género en edades tempranas y en el ámbito escolar es un objetivo señalado en las políticas nacionales de género en la Ley Orgánica 3/2007, de 22 de marzo (texto consolidado a 27/07/2013), para la Igualdad Efectiva de Mujeres y Hombres como vía para prevenir el problema endémico de violencia de género contra las mujeres. Expresamente se recoge entre los objetivos escolares el fomento de la igualdad plena entre mujeres y hombres con la eliminación de obstáculos que la dificultan y su tratamiento desde la idea de escuela coeducativa. Precisamente, una de las líneas de actuación prioritarias del I Plan de
Igualdad entre Hombres y Mujeres en Educación, puesto en marcha por la Consejería de Educación de la Junta de Andalucía en el año 2006 (actualmente vigente a la espera de la inminente aprobación del II Plan de Igualdad), pretende impulsar cambios en las relaciones de género con el fin de alcanzar una mayor igualdad entre mujeres y hombres. En este marco se recoge la obligación de las escuelas de desarrollar conocimiento diagnóstico de género por muy diversas vías; en este caso, se trata de desarrollar otro procedimiento más, complementario, que informa específicamente sobre el problema de la cohesión/distancia social de género, para completar el diagnóstico de género escolar.

Se apunta hacia la cultura y organización patriarcal (Bosch, Ferrer \& Alzamora, 2006) desde los organismos internacionales y nacionales, así como por las investigadoras e investigadores en los estudios de género y de las mujeres, como causa de la socialización desequilibrada de hombres y mujeres. También se ha señalado a la escuela como un contexto que favorece la reproducción social de las diferencias de género, lo que sólo puede contrarrestarse desde la coeducación (Subirats, 2011). El diagnóstico es clave para prevenir posibles conductas de riesgo, tales como la creación de relaciones desequilibradas basadas en actitudes sexistas y prejuicios de género, a la vez que para promover desde las escuelas medidas coeducativas que calen en las actitudes y las conductas del alumnado para que el cambio de la cultura patriarcal sea interiorizado y manifestado en las relaciones de igualdad entre chicos y chicas.

En este sentido, el objetivo principal de nuestro trabajo es aportar un conocimiento nuevo (patrones de preferencias relacionales de género) y una herramienta original (EPRG); válida, fiable y útil, para realizar este tipo de 
diagnóstico en materia de igualdad entre mujeres y hombres adolescentes. Por otro lado, este instrumento también sirve como recurso con el que contribuir a hacer visibles situaciones de actividad y prácticas relacionales de género transmisoras de los estereotipos sexistas patriarcales entre el alumnado adolescente, para intervenir en la cultura escolar tratando y eliminando aquellas situaciones que reproducen las desigualdades de género.

\section{Identidad de género, adolescencia y coeducación}

La construcción de la identidad de género se entiende como un proceso dinámico de interacción entre factores personales y sociales, que lleva a las personas a la asimilación, apropiación y reproducción de catalogaciones sociales diferenciales creencias, actitudes, comportamientos, expectativas, normas, roles y valores- según se sea mujer u hombre (Barberá \& MartínezBenlloch, 2004; Colás, 2007; Díaz-Martínez \& Dema, 2013; Martínez-Benlloch \& Bonilla, 2000; Subirats, 2011). Este establecimiento de pautas diferenciales asociadas a mujeres y hombres cobra sentido en el contexto cultural de relación entre las personas; dado que como se apunta desde el enfoque socio-conexionista "doing gender" (West \& Zimmerman, 1987), el género adquiere significado cuando se producen interacciones en contextos socioculturales (Crawford, 2006; Crawford \& Chaffin, 1997). Así, los hombres y las mujeres asumen e interiorizan la asignación social dicotómica de la identidad de género a través de la interacción con las personas (Barberá, 1998; Barberá, 2006).

Algunos estudios clásicos (Bosch et al., 2006; Lagarde, 1996; Lomas, 2007; Lorente, 2007; Lorente, 2009) han apuntado que la clave de la socialización de género está en la asignación diferencial a hombres y a mujeres de actividades y roles, de manera que se educa a chicos y chicas para que se comporten de formas diferentes y en ámbitos de actividad distintos, atribuyendo además un valor social desigual a lo masculino y lo femenino
(Rodríguez-Mosquera, 2011; Simón, 2010). Este proceso de socialización diferencial de género, que mujeres $\mathrm{y}$ hombres aprenden a través de la interacción con otras personas, reproduce los valores, las actitudes, las expectativas y los comportamientos sociales propios de su medio sociocultural para cada género. Esto ocurre como consecuencia del influjo de una serie de mediaciones subjetivas y culturales (el origen sexual, el lenguaje, la familia, la instrucción escolar, el grupo de iguales, el estatus económico y social, las ideologías, los estilos de vida, las creencias, los mensajes de la cultura de masas, etc.) que tienen su origen en la tradición patriarcal y que influyen de una manera determinante en la construcción de nuestras identidades.

Así, la socialización diferencial de género atribuye a las mujeres y los hombres ciertas características actitudinales y comportamentales estereotipadas que marcan y definen su identidad personal, y que son claramente opuestas entre sí (Simón, 2008; Tomé \& Tonucci, 2013). De esta manera, las mujeres constituyen su identidad de género con estereotipias tales como: la dependencia, la afectividad/sensibilidad, la comprensión, la empatía, la dedicación y el cuidado, la disposición a las demás personas, la sumisión, la pasividad, la tranquilidad, la belleza, la feminidad complaciente, etc.; mientras que los hombres configuran su identidad de género con estereotipias como: la independencia, la no emocionalidad/dureza, el individualismo, el egocentrismo, la competitividad, la centralidad en sí mismo, la asertividad, la dominancia, el riesgo, la fortaleza, la masculinidad normativa, etc. (Berk, 2009; Jensen-Arnett, 2008; Martínez-Sánchez, Navarro \& Yubero, 2009; Simón, 2010). Esta demarcación dicotómica entre los géneros no sólo se refiere a las actitudes y comportamientos de mujeres y hombres, sino que además se alude a la apariencia de género que se ofrece, materializándose ésta en la vestimenta y cuyos patrones sociales también son excluyentes entre sí: para las mujeres se espera una ropa llamativa, seductora y exclusivamente femenina sin posibles interpretaciones 
masculinas; y para los hombres, un atuendo sencillo, cómodo y claramente masculino (Zambrini, 2010). Estas atribuciones sociales de género influyen en la caracterización de las mujeres y los hombres, a la vez que predisponen el establecimiento de sus relaciones personales. Tanto es así que, dentro de este orden patriarcal dicotómico, también se establece un sistema binario en cuanto a la sexualidad: heterosexualidad/homosexualidad; naturalizando y normalizando la heterosexualidad, $\mathrm{y}$, por ende, silenciando $\mathrm{y}$ contemplando como excepcional la homosexualidad (Zambrini, 2013). De esta forma, la hombría o masculinidad hegemónica está caracterizada por la ausencia de feminismo y homosexualidad (Chaves, 2012; Piedra, 2013), al igual que la feminidad tradicional se concreta en la falta de masculinidad (Fernández-Sánchez, 2011).

La adolescencia es una etapa vital de gran relevancia en la construcción de la identidad de género (Berk, 2009; Jensen-Arnett, 2008; Morgade, 2009), ya que en ella tiene lugar gran parte de las transformaciones físicas, psíquicas y sociales o relacionales de las personas (García-Santesmases, Herrero, Olaso, Martínez-Ten, 2012; García-Tornel et al., 2011). Precisamente, recientes investigaciones revelan cómo la socialización diferencial de género ha calado en las actitudes y comportamientos de adolescentes, marcando especialmente las relaciones que establecen con sus iguales, las cuales reproducen desequilibrios interactivos según se trate de chicas o chicos y prácticas de riesgo, como actitudes sexistas, en la creación de vínculos (Bascón, Arias \& De la Mata, 2013; Ceballos, 2014; De la Peña, Ramos, Luzón \& Recio, 2011; Garaigordobil, 2012; García-Pérez et al., 2010; Herrera, Expósito \& Moya, 2012; Muñoz-Tinoco, Jiménez-Lagares \& Moreno, 2008; Ruiz-Pinto, García-Pérez \& RebolloCatalán, 2013; Soler, 2009).

Concretamente, De la Peña et al. (2011) desvelan que el sexismo en la adolescencia es más evidente, se hace más visible, en lo que se refiere a la asunción de roles de género -esto es: las tareas, funciones, actividades $\mathrm{y}$ responsabilidades propias asociadas a uno y otro sexo-, estando más interiorizada esta tradición sociocultural en los chicos que en las chicas adolescentes. En cuanto a las diferencias de género entre adolescentes en el plano relacional, Muñoz-Tinoco et al. (2008) observan que las chicas obtienen puntuaciones más altas en sociabilidad (ayudar, preocuparse, promover diálogos y acuerdos) y los chicos lo hacen en agresividad física y relacional (meter en líos a otras personas y manipular a los y las demás para conseguir sus objetivos). En la misma línea, Ruiz-Pinto et al. (2013) muestran, mediante análisis sociométrico de redes sociales (ARS), que en la adolescencia se elige más a las chicas para realizar tareas basadas en el cuidado y a los chicos para actividades de competición; mostrando además que las redes relacionales para el cuidado son más frágiles y dispersas, mientras que las relativas a la competición son más fuertes y cohesionadas. Soler (2009) estudia estos comportamientos estereotipados en las clases de Educación Física en el contexto escolar, encontrando que los chicos optan por compartir la actividad de jugar al fútbol con otros chicos de su mismo sexo y cuando participan chicas no las tratan con la misma consideración. Estos trabajos revelan la existencia de valores sexistas y de desequilibrio relacional entre chicas y chicos adolescentes, lo que lleva, ineludiblemente, a acciones desigualitarias entre los géneros y a posibles prácticas de riesgo como episodios de violencia de género (Bonino, 2005; Ferrer, Bosch, Navarro, Ramis \& García, 2008; Lorente, 2007), así como a la justificación del uso de la violencia, tanto por parte de chicas como de chicos (Garaigordobil \& Aliri, 2013). Es por ello, que cobra una especial importancia educar en un modelo sociocultural que lleve por principio la igualdad entre las personas para facilitar un cambio en las actitudes, los comportamientos y las relaciones de chicos y chicas adolescentes.

A la luz de los resultados de estas investigaciones, es evidente la clara necesidad de intervención educativa con el fin de 
transformar el imaginario social y de facilitar nuevos modelos de masculinidad y feminidad, cuyos principios centrales sean la igualdad, el respeto y la pluralidad. El carácter eminentemente interactivo de la escuela, así como de la propia estructura educativa: educador/a, educando/a y contenidos, ha propiciado tradicionalmente la reproducción de desigualdades socioculturales hacia las mujeres y los hombres por razón de género (Bueno \& Garrido, 2012); no obstante, y, precisamente, por esta cualidad interactiva de la educación, una escuela que se asienta en una perspectiva integral y de género, lleva a favorecer la construcción de la igualdad y a mejorar la convivencia, a la vez que prevenir cualquier tipo de violencia, incluida la violencia de género (Díaz-Aguado \& MartínSeoane, 2011).

Una intervención educativa, que tenga como pilar central la igualdad entre géneros y la no discriminación por razón de sexo, requiere del conocimiento exhaustivo de las situaciones de actividad y contextos relacionales del alumnado en que se producen mayores distancias sociales y falta de cohesión inter-intra géneros. Este conocimiento facilita la intervención en un sistema coeducativo (Ferrer \& Bosch, 2013; Simón, 2010; Subirats, 2009; Subirats \& Tomé, 2007), el cual ha de aspirar a la construcción de nuevas identidades de género, libres de estereotipos sociales y relaciones desequilibradas entre chicas $y$ chicos, con el propósito final de conseguir la igualdad y reconocer las diferencias propias de cada género (Meseguer \& Villar, 2011; Subirats, 2010).

\section{Preferencias Relacionales de Género: un nuevo sistema de diagnóstico en educación}

Arteaga y Lara (2004) nos ofrecen una panorámica muy interesante para situar la aproximación metodológica de las escalas de preferencias relacionales en el marco de los esfuerzos vinculables al desarrollo de conocimientos “sociológicos” y su derivación hacia planteamientos educativos $\mathrm{y}$ transformadores de la sociedad actual y la violencia de género. Dicha violencia de género se manifiesta desde los primeros años de la vida humana actual y la escuela es, sin duda, el lugar idóneo donde estudiarlo para conocerlo bien y poder atajarlo. Como señala Arteaga (2003), la sociología se ha venido desarrollando desde su origen en un continuo viaje, de ida y vuelta, entre los determinantes estructurales y la acción individual.

El enfoque que guía la creación de las "escalas de preferencias relacionales" se basa en la superación de contrarios que implica 1) el enfoque estructuralista de Moreno (1934), que ya hemos practicado en este mismo contexto bajo la idea del novedoso "Análisis de Redes Sociales” con UCINET (Ruiz-Pinto et al., 2013), y 2) la aproximación de Bogardus a la medida del constructo "Distancia Social" con origen en Durkheim (Collins, 1974), ambos son enfoques que tratan de abordar el problema de la cohesión social, o mejor la falta de ella, como elemento clave en el que fermenta "la violencia y la agresión" de los considerados "contrarios u opuestos" y la negación para estos del cambio y la solidaridad hacia la igualdad; una negación que se basa en la "normalización” del rechazo y la violencia como parte de las relaciones naturales (o mejor socialmente naturalizadas) en el ámbito de la convivencia social.

Queremos, pues, realizar una "microsociometría educativa” nueva, que no sólo nos indique en sentido diagnóstico dónde están los problemas, sino que apunte por sí misma y por la información que ofrece hacia la manera de solucionarlos. Para ello, tanto las aproximaciones estructurales (con sus análisis de cohesión y centralidad a partir de la aceptación y el rechazo del otro) como las aproximaciones de las escalas de distancia social hacia el “otro grupo” (según, raza, etnia, nacionalidad, género, clase social, orientación sexual, afectados por problemas económicos o de salud, etc.), permiten un punto metodológico intermedio que nos muestre las “clases” y "categorías" de la aceptación y el rechazo en un modelo nuevo e integrado de interpretación. Por ello, las "Escalas de Preferencias Relacionales de Género” (EPRG) 
que hemos formulado se nutren tanto de la fórmula clásica del test sociométrico iniciado por Moreno (1934) como de las escalas de distancia social de Bogardus; constituyendo un enfoque metodológico interdisciplinar nuevo y útil, muy específicamente, al problema que hemos planteado. Este nuevo enfoque supone considerar a un tiempo tanto la actitud relacional y el "tele" intersubjetivo como la disposición a la interacción concreta y específica según los diversos "entornos claves de relación” de los que la teoría informa como conflictivos, en este caso, en lo que respecta a preferencias relacionales de género y mandatos del patriarcado.

Entre estos elementos teóricos que informan la estructura de una escala de preferencias relacionales de género han de considerarse especialmente los conocidos elementos vinculados al sostén del patriarcado (Guasch, 2007) tanto de diferenciación de patrones sexuales y de presupuestos estereotipados sobre el género como los elementos claves relativos a la homofobia, pues, precisamente el propio Bogardus (1965) indicaba que el miedo es el factor más relevante y dinámico en la producción causal y mantenimiento de la distancia social. El no cumplimiento de los patrones patriarcales asignados a los varones (cuyas identidades han de ser construidas en base a los estereotipos sexuales heteronormativos) para la dominación del mundo social y, de los patrones de función social justificada en los cuidados y en el marco de lo íntimo para las mujeres, supone pues la base del "miedo al desencaje social" patriarcal transmitido desde la propia familia a las personas en la más tierna infancia.

Este tipo de escalas suponen variantes de los tests sociométricos en las que no preguntamos expresamente por cada persona y su aceptación o rechazo, sino que preguntamos por la aceptación o rechazo del grupo social (considerados como "objetos" completos e irreductibles, no persona a persona; por ejemplo, en este caso preguntamos a los chicos y las chicas que acciones conjuntas llevaría o no a cabo con otros "chicos" y/o "chicas" de su propia aula en determinados contextos de actividad teóricamente propuestos).

Este tipo de instrumentación y análisis, como hemos señalado antes, puede usarse en el marco de cualquier propuesta de análisis de la "distancia socio-estructural” de los grupos que puedan formarse según variaciones en la identidad, el status y las brechas culturales e intergeneracionales. Las respuestas a las escalas de preferencias relacionales (sean estas de carácter métrico o no métrico -en nuestro caso son variables categóricas en escala nominal-) se analizan en sentido multivariante (por ejemplo aplicando Análisis de Correspondencias Múltiples y Análisis de Clusters) para poderlas representar bajo un mismo espacio multidimensional en las que las "distancias sociales" investigadas se manifiestan bajo la idea de "distancia espacial geométrica” (Cornejo, 2003b; 2006), indicando lo elementos que son claves para la intervención educativa y la prevención sociocultural en muy diversos planos que afectan a la educación. Esta concepción de la aproximación espacial a las distancias sociales tiene apoyo en las propuestas metodológicas de Cornejo (1988; 2003a) para detectar la proximidad, igualdad o semejanza a partir del Análisis de Correspondencias Múltiples de las interacciones entre personas y de grupos.

\section{Objetivos}

El objetivo principal del estudio es valorar las preferencias relacionales de género del alumnado, dentro del proceso de diagnóstico de género en las escuelas, con el propósito de intervenir sobre la cultura escolar para prevenir, desde edades tempranas, los desequilibrios y consecuencias de una socialización de género muy diferenciada y extrema. Esto es, necesitamos reconocer los patrones preferenciales en las relaciones de género, para prevenir futuras conductas de riesgo en el plano relacional, en cuanto a violencia física, psicológica o de microviolencia. La microviolencia o los micromachismos (Bonino, 2005; Ferrer et al., 2008) son formas culturales de dominación que también se manifiestan en la organización 
escolar (Castells \& Subirats, 2007; Subirats \& Tomé, 2007). Valorar estos patrones generales que se detraen del conjunto de datos con la aplicación de estas técnicas sociométricas híbridas nos sirven, en última instancia, para proponer ideas útiles para la coeducación y la transición desde la escuela mixta a la escuela verdaderamente coeducativa.

Por tanto, desglosamos esta meta global en dos objetivos más concretos y específicos que dan el valor al estudio realizado:

1. Validar la Escala de Preferencias Relacionales de Género (EPRG) que hemos usado para descubrir y valorar los patrones relacionales entre chicos y chicas en el marco de las aulas escolares; esto es, más precisamente, validar las medidas con ellas obtenidas en este estudio concreto. La consecución de este objetivo implica no solo obtener unos datos válidos y fiables para resolver nuestro estudio sino también, complementariamente, proponer a la comunicada educativa un nuevo sistema para desarrollar este tipo de métrica socioeducativa de género.

2. Reconocer y explicar los patrones relacionales de género en la escuela andaluza, en un sentido multivariante, a partir de las respuestas del alumnado a la Escala de Preferencias Relacionales de Género (EPRG); identificando y diferenciando los principales patrones asociados a hombres y mujeres, relativos tanto a la aceptación como al rechazo para los colectivos de chicos y chicas. De esta forma, se completa el objetivo global de aportar una perspectiva sociológica de la educación dentro del proceso global de diagnóstico de género en el ámbito escolar.

\section{Método}

Se desarrolla un estudio correlacional multivariante, basado en el Análisis de Correspondencias Múltiples de los datos procedentes de las Escalas de Preferencias Relacionales de Género (EPRG) con el objetivo de analizar relaciones de género en la adolescencia y, específicamente aplicar esta nueva técnica diagnóstica para valorar la predisposición del alumnado a elegir/rechazar chicos y/o chicas en función de la naturaleza de las actividades desvelando sus atribuciones de roles de género y estereotipos.

\section{Participantes}

Participan en este estudio 6000 alumnos (49,2\%) y alumnas (50,8\%), de 56 centros de enseñanzas formales varias (primaria, secundaria, FP y Bachillerato) que cursan estudios en centros públicos y concertados de Andalucía (España) durante el curso académico 2009-2010, siendo la muestra fundamentalmente adolescentes de secundaria (74,8\%); la edad media es de 13,82 años (mediana $=14$; d.t. $=2,42)$; correspondiendo a diferentes rangos de edad $(<13=31 \%$; $13-14$ $=32,9 \% ; 15-16=25,9 \% ; 17-18=7,4 \% ;>18$ $=2,8 \%$ ), de los cuales un 57,2\% proviene de zona urbanas y el $42,9 \%$ no urbanas. Se aplica un muestreo por conglomerados (aulas) de centros de la educación pública andaluza, contemplando criterios de inclusión según niveles educativos, zona geográfica y ámbito sociocultural. 


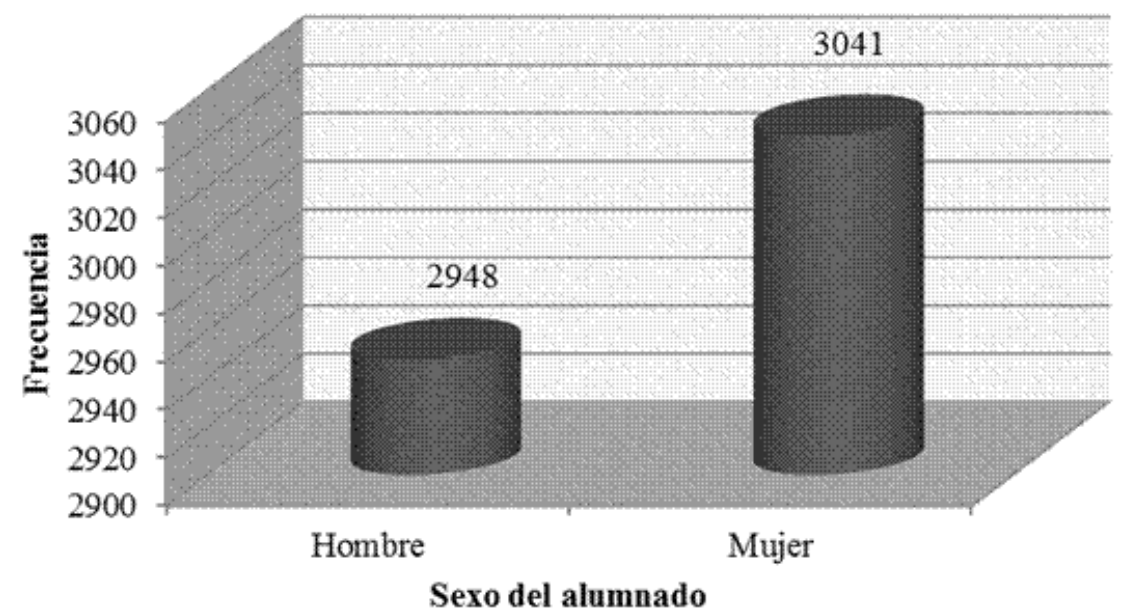

Figura 1. Datos sociodemográficos de la muestra (sexo del alumnado)

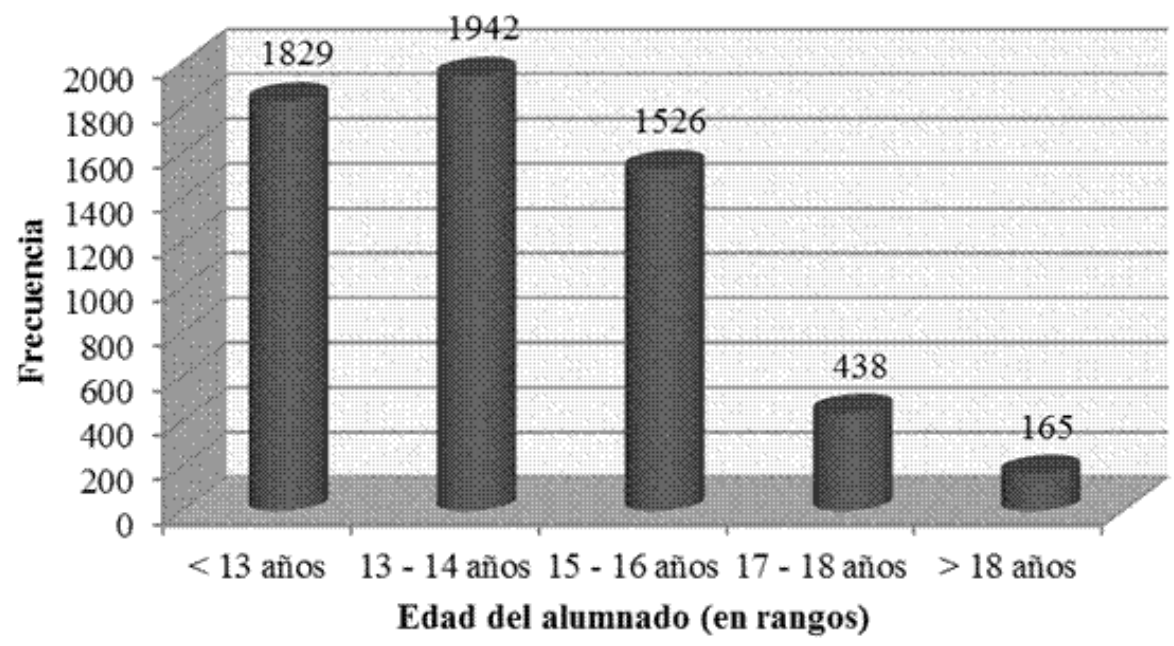

Figura 2. Datos sociodemográficos de la muestra (edad del alumnado expresada en rangos).

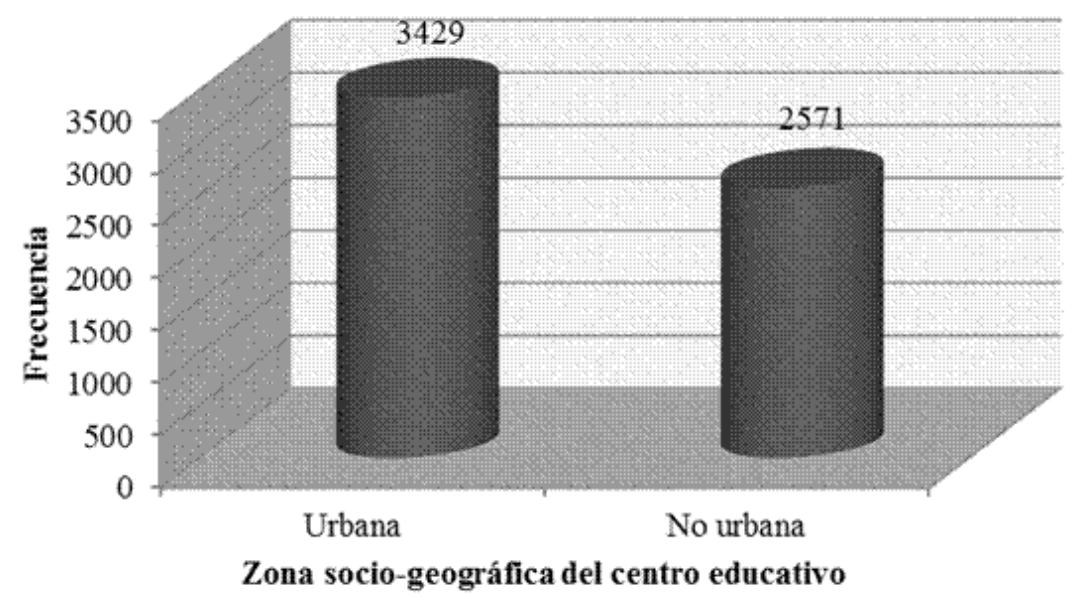

Figura 3. Datos sociodemográficos de la muestra (zona socio-geográfica del centro educativo). 


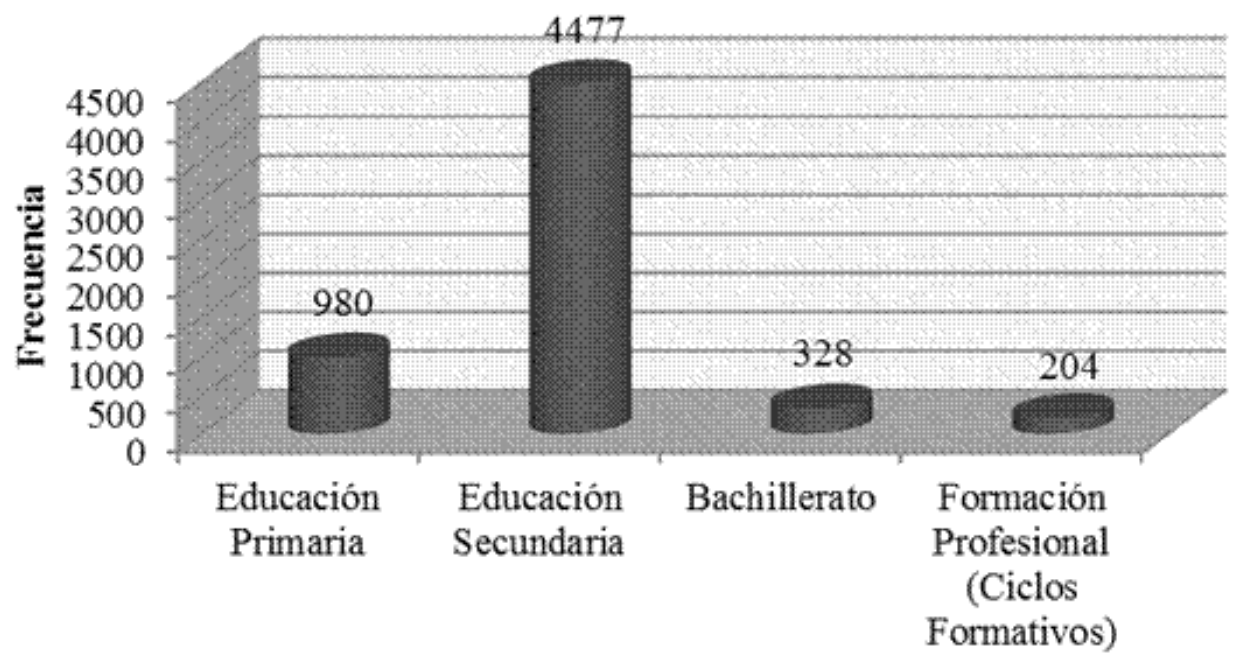

Nivel formativo del alumnado

Figura 4. Datos sociodemográficos de la muestra (nivel formativo del alumnado).

\section{Instrumento}

La técnica de encuesta utilizada se articula mediante un cuestionario que recoge, junto a los usuales datos sociodemográficos y de procedencia que nos han permitido caracterizar la muestra anterior, la Escala de Preferencias Relacionales de Género (EPRG). Ya se ha señalado en la aproximación conceptual que estas constituyen un tipo de instrumento sociológico nuevo o "híbrido" entre las escalas de distancia social de Bogardus y los test sociométricos clásicos propuestos por Moreno (1934), cuyo sentido teórico y utilidad científica se ha justificado anteriormente. Ahora, en este apartado, procedemos a exponer su naturaleza metodológica y a presentar la escala EPRG construida para este trabajo específico (véase figura 5).

Como se observa, esta escala se compone de un conjunto de ítems dicotómicos (si/no) referidos a contextos y situaciones concretas en posibles actividades relacionales de género entre los chicos y las chicas del aula escolar, agrupados según dimensiones que se repiten para cada género. Por tanto, se incluyen cuatro dimensiones (dos para valorar las preferencias relacionales con los chicos y otras dos para las chicas) dentro de las cuales se encuentran los diez ítems que dimensionan cada una. Con estos 40 ítems se constituye una matriz de respuestas sociométrica, pues cada par de dimensiones dirigidas a un género se refiere a la aceptación o rechazo de los chicos y de las chicas, según dimensión, en relación con los diez contextos de acción relacional/comunicativa que se proponen a evaluación. Estos contextos relacionales son:

1. Tener una relación de pareja

2. Estudiar juntos/as

3. Compartir un secreto

4. Sentarnos juntos/as en la clase

5. Prestarnos la ropa

6. Ordenar y limpiar

7. Practicar deporte juntos/as

8. Jugar a un videojuego

9. Ser delegado/a de la clase

10. Ir de acampada 


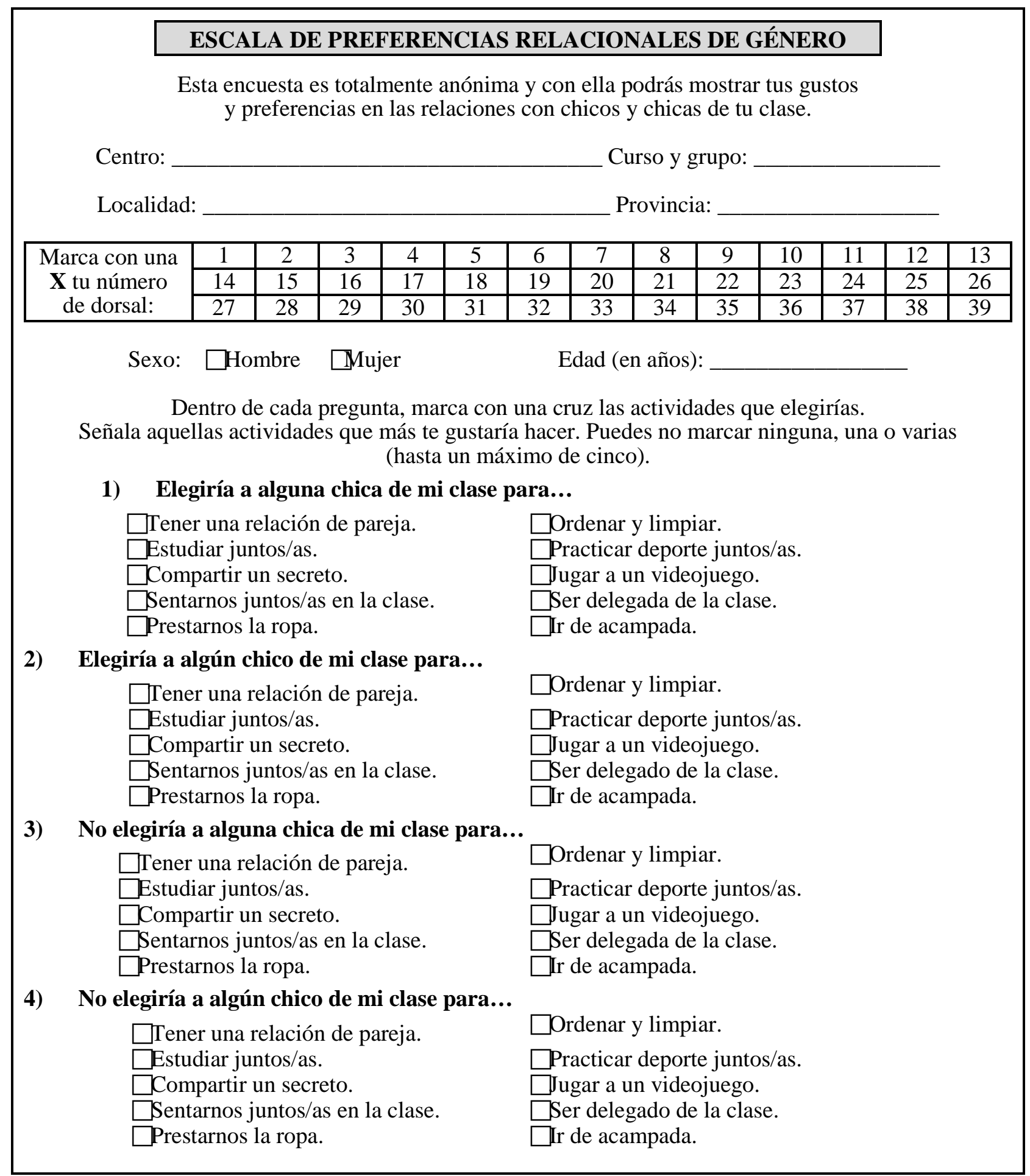

\section{Figura 5. Escala de Preferencias Relacionales de Género (EPRG)}

De esta forma, la primera dimensión recoge la aceptación del género femenino (chicas) tanto por chicos como chicas (esto es, por el conjunto de la muestra de 6.000 hombres y mujeres) para el desarrollo de la actividad relacional que propone cada uno de los 10 ítems. La segunda dimensión de forma análoga recoge la aceptación del género masculino para las mismas actividades $\mathrm{y}$, también, por toda la muestra. Igualmente, las dimensiones tercera y cuarta se dedican de forma idéntica a recoger las preferencias relacionales, pero en sentido de rechazo, ya no de aceptación.

Es decir, se recogen datos que expresan tanto las aproximaciones de los tests 
sociométricos para la identificación de elecciones (aceptación y rechazo), pero vinculados a los géneros (al estilo de las distancias sociales de "contrarios y opuestos" de Bogardus). De esta forma se constituye la matriz de preferencias relacionales con 40 dimensiones que son óptimas para desarrollar escalamientos basados en la aplicación de Análisis de Correspondencias Múltiples.

\section{Procedimiento}

Inicialmente, se presentó el estudio y se propuso la participación en el mismo a los equipos directivos de los centros educativos andaluces, acordando con éstos que la aplicación de la Escala de Preferencias Relacionales de Género (EPRG) al alumnado se realizaría de forma colectiva a cada grupoclase, en menos de 50 minutos dentro del horario lectivo semanal dedicado a tutorías en cada grupo-clase, y contando con la colaboración del profesorado-tutor/a de cada grupo-clase. Al alumnado se le informó de la utilización que se haría de los datos recogidos, así como del carácter voluntario y anónimo de su participación en el estudio.

Los datos han sido procesados con el paquete estadístico SPSS (versión 20 para Windows) y, posteriormente, analizados con el programa SPAD (versión 5.6 para Windows), con el cual se han realizado los análisis de correspondencias múltiples, siguiendo los planteamientos de Cornejo (2003a), Joaristi y Lizasoain, (1999) y Sánchez Carrión, (1989).

\section{Resultados}

\section{Validación de las medidas obtenidas con la Escala de Preferencias Relacionales de Género (EPRG)}

No hay mucha tradición de análisis de la calidad de los datos mediante este tipo de escalas que proponemos, por lo que hemos buscado un análisis similar en la propuesta de Bergesio y Golovanevsky (2008; 2013) y de Letelier et al. (2010). El estudio de la validez y fiabilidad para el caso de datos dicotómicos (elección/no elección y rechazo/no rechazo) obtenidos con las escalas de preferencias relacionales y organizados mediante matrices de datos nominales, tal como se presenta en este estudio, requiere de un análisis pormenorizado de los valores obtenidos en el modelo de correspondencias múltiples que se considere como válido para la observación y graduación "geométrica” de las posiciones espaciales en el nuevo espacio euclideo reducido a los componentes principales que pueden mantener una explicación parsimoniosa pero precisa del problema de diagnóstico que se presenta. Esto quiere decir que la validez de constructo alude aquí al hallazgo de un modelo de Análisis de Correspondencias Múltiples (ACM) útil para representar la información con los menores componentes principales posibles pero que esta se pueda observar de forma nítida en relación con la naturaleza del problema de investigación.

Tal es el caso de este modelo de ACM que presentamos (tomando como variables activas las 40 dimensiones -10x4- que contempla el instrumento más la variable género). De esta forma, se busca un modelo útil para observar las asignaciones de roles de género $\mathrm{y}$ estereotipos (según el problema planteado en el estudio) que a su vez es parsimonioso, pues sólo requiere conservar para la explicación los dos primeros factores, pues a partir del tercero ya sólo se explican aspectos residuales, principalmente redundancia de información contenida en los dos primeros y que tienen una peor representación espacial.

\begin{tabular}{|c|c|c|c|c|}
\hline NUMBER | EIGENVALUE | PERCENTAGE | CUNULATED | \\
|
\end{tabular}

Figura 6. Extracción de los 5 primeros componentes principales en el ACM 
Una vez resuelto el problema de hallar el modelo adecuado de ACM, es necesario comprobar que la nitidez con que se representan, conjuntamente, los casos y variables, en el espacio euclideo así caracterizado, es suficiente (fiable). Para ello, contamos con la generalización del teorema del coseno en el espacio euclideo, que nos da como resultado que los "cosinus carres/squares cosines" (cosenos al cuadrado) dan cuenta de la potencia representativa de un elemento en el espacio vectorial elaborado con el modelo de ACM elegido. Es decir, que en la práctica estos son indicadores, a modo de coeficientes de correlación entre las dimensiones y las variables, sobre la nitidez de observación del elemento (categorías de las variables) en cada plano geométrico en que se impliquen tales dimensiones (ejes 1 y 2 2). En nuestra investigación, la nitidez que ofrece a las distribuciones de los roles de género la dimensión
2 (que se elabora con la contribución principal del género de los encuestado) alcanza un coeficiente muy elevado (Squares Cosines $=.71=$ Hombre/Mujer). De donde se deduce que las observaciones de las diferencias de género (distancias), esencia de este estudio, sobre los planos conformados por los ejes 1 (pues este representa bien las elecciones/rechazos) y 2 serán claramente fiables y pueden conducir a una interpretación científica útil.

Exponemos solamente los valores de las categorías de género (hombre/mujer) y los 10 primeros elementos categóricos (si/no) referidos a las variables del ítem 1 de la escala de preferencias relacionales de género EPRG para no caer en redundancias porque los restantes ítems siguen exactamente la misma lógica y no ofrecen sino idéntica información a la aquí expuesta.

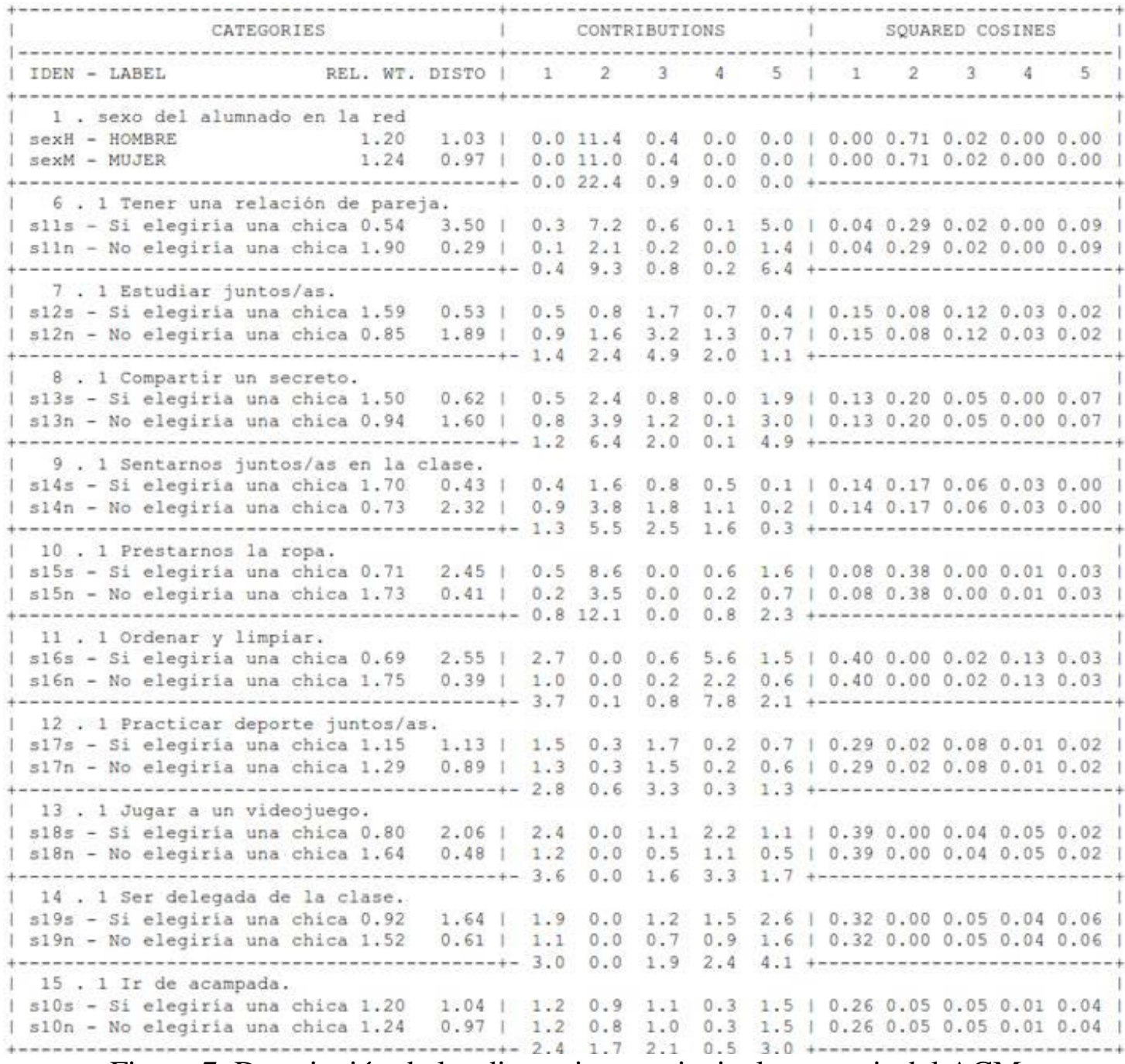

Figura 7. Descripción de las dimensiones principales a partir del ACM 
Además, para las aproximaciones de las variables no activas (ilustrativas) contamos con los valores $t$ (que indican distancias en valores positivos y negativos normalizadas respecto del centro del factor o dimensión) que se ajustan mediante una prueba de Chi-2 indicativa de su significación en la definición y explicación de su asociación con cada factor y/o cluster calculado. Si bien, como se verá posteriormente, el análisis gráfico viene a ser aún más expresivo sobre los planos principales que conforman ambos ejes, por lo que no se incluyen en el documento.

Complementariamente, hemos calculado el coeficiente Alpha de Cronbach para el conjunto de la escala EPRG (con el procedimiento reliability del SPSS) obteniendo un óptimo resultado en consistencia interna (Alpha $=.92)$, lo cual era de esperar dado los marcados que están los roles de género que promueve el patriarcado en los procesos de socialización de chicos y chicas (ambos son muy consistentes tanto en la elección como en el rechazo promoviendo altos niveles de concordancia grupal entre sí), manifestando que este tipo de aproximación diagnóstica novedosa que suponen las escalas de preferencias relacionales de género ofrecen una clara caracterización del problema de estudio abordado en el diagnóstico de género en los centros escolares. También es justo decir, que el tamaño muestral es muy amplio ( $\mathrm{n}=6000$ casos; 2948 chicos, 3041 chicas -y 11 casos que no determinan su género-) lo que afecta a la elevación de este coeficiente. Finalmente, para no incumplir supuestos de nivel de medida, hemos considerado el caso del cálculo de un modelo de escalamiento óptimo (CATPCA para datos nominales con categorías múltiples no ordenadas -en nuestro caso para las 40 variables de la escala EPRG cada categoría toma los valores $1=\mathrm{si}, 2=$ no y $3=$ ns/nc; y el género 1=mujer, 2=hombre $\mathrm{y}$ $3=\mathrm{ns} / \mathrm{nc}$ ) obteniendo como resumen un modelo de dos factores principales en el cual el género satura obteniendo medidas de discriminación altas en la segunda dimensión (.707; con una consistencia Alpha de Cronbach $=.701$ ) y las variables de aceptación/rechazo saturan generalmente con mayores valores en la dimensión 1 (con un Alpha = .93).

Resumen del modelo

\begin{tabular}{|c|c|c|c|c|c|c|c|}
\hline \multirow{2}{*}{ Dimensión } & \multirow{2}{*}{$\begin{array}{c}\text { Alfa de } \\
\text { Cronbach }\end{array}$} & \multicolumn{2}{|c|}{ Varianza explicada } & & & & \\
\hline & & Total (autovalores) & Inercia & \multicolumn{4}{|c|}{ Medidas de discriminación } \\
\hline 1 & ,930 & 10,748 & ,262 & \multicolumn{4}{|c|}{ (sólo se expone la variable género) } \\
\hline 2 & ,701 & 3,162 & ,077 & \multirow{3}{*}{$\begin{array}{l}\text { Sexo del } \\
\text { alumnado en } \\
\text { la red }\end{array}$} & Din & sión & \multirow{2}{*}{ Media } \\
\hline Total & & 13,910 & ,339 & & 1 & 2 & \\
\hline Media &, $878^{\mathrm{a}}$ & 6,955 & 170 & & ,000 & ,707 & ,354 \\
\hline
\end{tabular}

a. El Alfa de Cronbach Promedio está basado en los autovalores promedio

Figura 8. Fiabilidad de las mediciones categóricas y discriminación por género.

Considerando el conjunto de resultados dispuestos a discusión, cabe interpretar que la medida obtenida en el proceso de recogida de datos socioeducativos con la Escala de Preferencias Relacionales de Género (EPRG) es una aproximación bastante fiable (consistente) y válida (constructo: asignaciones de roles y estereotipos de género en el aula escolar) considerando al tiempo las “distancias sociales” y la estructura electiva de chicos y chicas en las aulas. Con esta confianza procedemos en el siguiente apartado al objetivo de caracterizar las preferencias relacionales de género.

\section{Patrones relacionales de género de chicas y chicos en el ámbito escolar}

Una vez validado el procedimiento novedoso de análisis sociométrico, hemos indicado que nuestro objetivo era reconocer y 
explicar los patrones relacionales de género que mostraban, los chicos y las chicas adolescentes en los contextos escolares, a través de sus elecciones/rechazos, en el marco de situaciones relacionales concretas; siguiendo la lógica del nuevo procedimiento de medición.

Por tanto, una vez obtenidas estas elecciones y rechazos, a partir de las respuestas del alumnado a la Escala de Preferencias Relacionales de Género (EPRG); se han analizado las contribuciones a las dimensiones obtenidas en el ACM, identificando los factores 1 y 2 como los principales a estudiar.

Se continua diferenciando los principales patrones asociados a hombres y mujeres, a partir de las inter-distancias que se constatan en el nuevo espacio métrico generado por el plano de los ejes 1 y 2 , lo que se repite tanto para chicos como para chicas (a efectos de visibilidad) y relativos tanto a la aceptación como al rechazo (figuras 9, 10, 11 y 12) para ambos colectivos de género. Se presentan a continuación:

Figura 9. Distribución de las Preferencias Relacionales de Género de mujeres y hombres adolescentes en la elección de chicas para supuestos de interacción

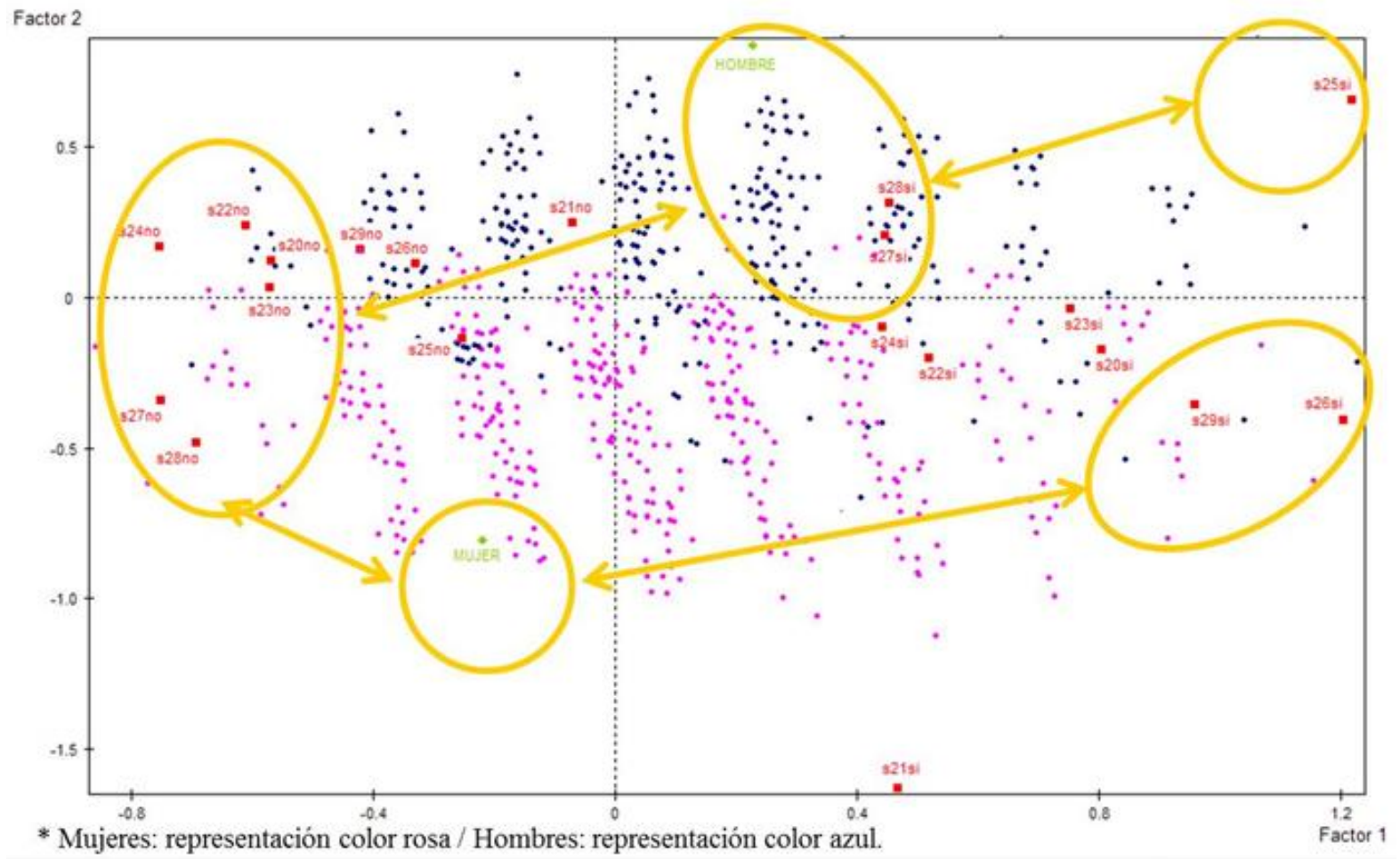

Figura 10. Distribución de las Preferencias Relacionales de Género de mujeres y hombres adolescentes en la elección de chicos para supuestos de interacción

Estos dos primeros planos recogen la aceptación de chicos y chicas, indicando las preferencias relacionales en positivo, lo que se tiene en cuenta para la interpretación. 


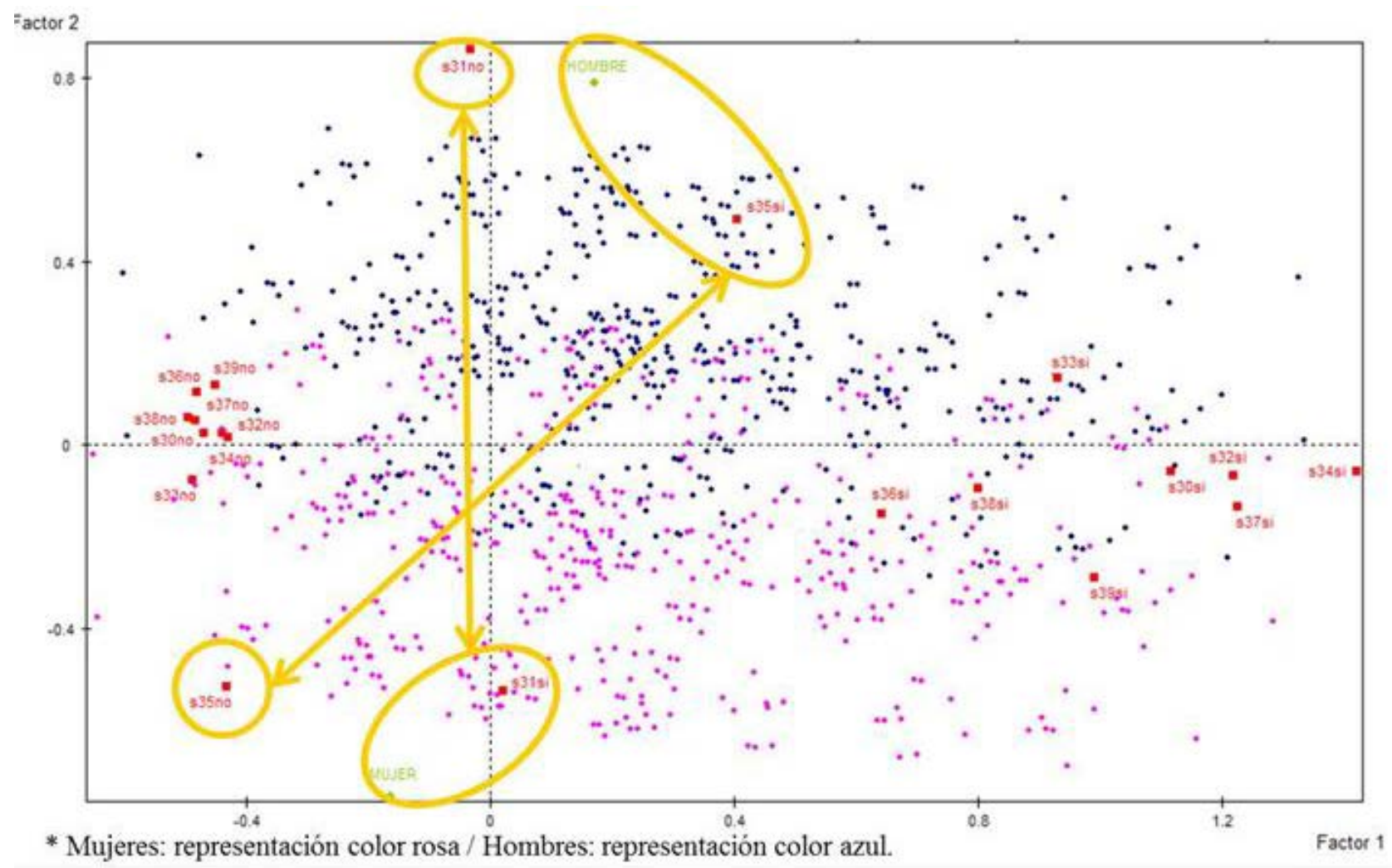

Figura 11. Distribución de las Preferencias Relacionales de Género de mujeres y hombres adolescentes en el rechazo de chicas para supuestos de interacción

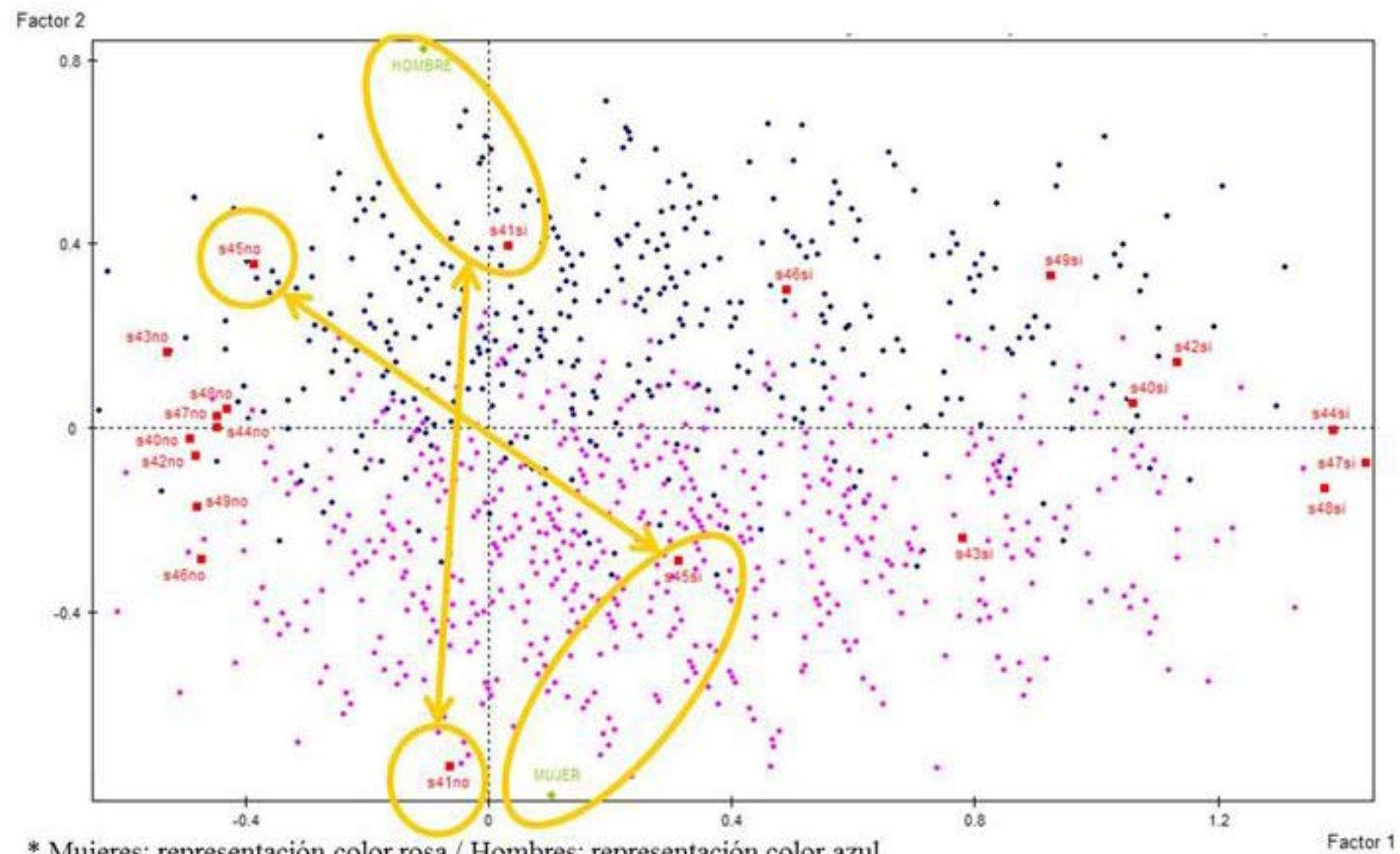

* Mujeres: representación color rosa / Hombres: representación color azul.

Figura 12. Distribución de las Preferencias Relacionales de Género de mujeres y hombres adolescentes en el rechazo de chicos para supuestos de interacción 
Este segundo par de planos recogen el rechazo de chicos y chicas, indicando las preferencias relacionales en negativo, lo que se tiene en cuenta para la interpretación.

Considerando toda la información cuantitativa ofrecida por los factores y categorías en su relación con las dimensiones y las representaciones gráficas expuestas en las últimas figuras (figuras 9, 10, 11 y 12), podemos valorar los principales rasgos de los patrones relacionales de género entre adolescentes. De manera, la figura 9 recoge las preferencias relacionales de mujeres y hombres para interaccionar con las chicas. Sobre esta ilustración gráfica hemos marcado las relaciones. Así, se puede observar como los hombres de la muestra eligen mayoritariamente a chicas para tener una relación de pareja [s11si]; mientras que las mujeres de la misma muestra optan por elegir a chicas para compartir un secreto [s13si], sentarse juntas en la clase [s14si] y estudiar juntas [s12si].

Por su parte, la figura 10, presenta las elecciones que los hombres y las mujeres de la muestra realizan para actividades relaciones con chicos. Los hombres prefieren relacionarse con chicos para jugar a un videojuego [s28si], practicar deportes juntos [s27si] y prestarse la ropa [s25si]. Las mujeres escogen a los chicos para diversas actividades, aunque no destaca mayoritariamente ninguna elección significativa.

En el plano de la negación de relaciones, las figuras 11 y 12 reflejan las preferencias relacionales de género en cuanto al rechazo explícito de chicas y chicos, respectivamente. Así, cabe destacar que tanto en la figura 11 como en la figura 12 se aprecia la misma tendencia de rechazo relacional en las mujeres y en los hombres. En la figura 11, las mujeres de la muestra manifiestan su clara negativa a tener una relación de pareja con otra chica [s31si] y los hombres a prestarse la ropa con una chica [s35si]. De la misma manera, en la figura 12 la predisposición relacional es idéntica pero a la inversa: las mujeres rechazan la relación con chicos para prestarse la ropa [s45si] y los hombres no quieren relacionarse con chicos para tener una relación de pareja [s41si].

De esta forma, vemos como técnicamente hemos dibujado los patrones del patriarcado sobre la base de las respuestas múltiples ofrecidas por la muestra de adolescentes. Pero, quizás, lo más significativo de este análisis sea que podemos identificar y diferenciar los posicionamientos individuales de cada caso (persona), sea hombre o mujer, estableciendo su distancia o cercanía con los patrones aquí dibujados. Con ello, establecemos una base para el diagnóstico de la propia identificación personal o des-identificación particular que cada cual muestra respecto de los patrones patriarcales teorizados y empíricamente mostrados con la Escala de Preferencias Relacionales de Género.

\section{Discusión y conclusiones}

A modo de discusión con otros estudios, los resultados en este trabajo corroboran la construcción de identidades de género diferenciales entre chicos y chicas, así como las estereotipias de género patriarcales (Barberá \& Martínez-Benlloch, 2004; Berk, 2009; Colás, 2007; Díaz-Martínez \& Dema, 2013; Martínez-Benlloch \& Bonilla, 2000; Subirats, 2011; Tomé \& Tonucci, 2013). Esto se demuestra tanto para las actitudes generales inter-intra géneros como las actitudes vinculadas a los contextos de actividad relacional y conductas concretas. De esta forma, la confianza y otros elementos relativos a los cuidados y atenciones con los demás hacen que las chicas sean más elegidas, siendo los chicos preferidos para actividades competitivas, lo que corrobora lo establecido en anteriores investigaciones (Ruiz-Pinto et al., 2013). En la elección inter-intra géneros para las relaciones sentimentales de pareja se confirma el patrón patriarcal heteronormativo dominante (Chaves, 2012; Fernández-Sánchez, 2011; Piedra, 2013; Zambrini, 2013). Igualmente se afirman las identidades intragénero, opuestas a modas andróginas, haciéndolas muy manifiestamente diferenciadas (como para que no puedan 
confundirse) en actividades y contextos relacionales tales como la ropa que se viste (Zambrini, 2010) y/o la elección de pareja (Guasch, 2007); donde se muestra el miedo a la indiferenciación/confusión sexual indicando al patrón homofóbico patriarcal. De todo ello se detrae la necesidad de profundizar en aquellos enfoques que patrocinan la coeducación y la libre diferenciación de la identidad personal desde la propia escuela (Meseguer \& Villar, 2011; Simón, 2010; Subirats \& Tomé, 2007).

$\mathrm{Si}$ bien estos resultados confirman lo establecido en la teoría de género, se han investigado con una técnica sociométrica novedosa, las escalas de Preferencias Relacionales de Género (EPRG), que muestra altos indicadores de validez y fiabilidad, aportando una información distinta a otros procedimientos sociométricos que ya hemos ensayado con base en este mismo problema de investigación: patrones relacionales de género entre el alumnado (Ruiz-Pinto et al., 2013). Esta nueva aproximación que aquí hemos probado se diferencia de otras sociometrías más clásicas porque expresa los patrones sociales de aceptación/rechazo y cohesión/distancia desde un específico vínculo con los "contextos y situaciones relacionales". Ello supone un plus informativo que posibilita matizar las diferencias en los patrones sociales, indicando las áreas, temáticas y contextos relacionales concretos en los que la "fricción" o desentendimiento se hace más presente, aportando un conocimiento tan específico de ello que podríamos denominarlo como "micro-sociométrico". Por tanto, este enfoque es útil para aproximarse a una sociometría que pueda poner de manifiesto y tal vez explicar en un sentido causal dinámico las microviolencias de género a que se refieren Bonino (2005) y Ferrer et al. (2008).

De otro lado, este estudio aporta una especificación de los patrones patriarcales de género que afectan a nuestro alumnado adolescente, estudiando con técnicas correlacionales multivariantes una amplia muestra, para definir un espacio métrico social que se configura empíricamente desde las respuestas de aceptación/rechazo de los sujetos, indicando la posición de cada sujeto, categoría y grupo sobre dicho espacio analítico. De esta forma se obtiene un mapa de interdistancias que no sólo dibuja y permite manifestar los patrones relacionales de género, claramente vislumbrados en el apartado último de resultados, sino que se revela la existencia de múltiples posicionamientos individuales (manifestando así la diversidad) que no siempre responden al patrón establecido o exactamente esperable tanto para chicos como para chicas. Desde esta propiedad o aportación de esta técnica puede trabajarse en la identificación de casos típicos/atípicos para estudios más específicos y/o cualitativos, como los que se aplican para el estudio de las nuevas identidades de género.

\section{Referencias}

Arteaga, N. \& Lara, V.L. (2004). Violencia y distancia social: una revisión. Papeles de Población, 10(40), 169-191. Recuperado de: http://www.redalyc.org/articulo.oa?id=11204010

Arteaga, N. (2003). El espacio de la violencia: un modelo de interpretación social. Sociológica, 18(52), 119-145. Recuperado de: http://www.revistasociologica.com.mx/pdf/5205. pdf

Barberá, E. (1998). Psicología del género. Barcelona: Ariel.

Barberá, E. (2006). Aportaciones de la psicología al estudio de las relaciones de género. En C. Rodríguez-Martínez (Comp.). Género y currículo: aportaciones del género al estudio y práctica del currículo (pp. 59-76). Madrid: Akal.

Barberá, E. \& Martínez-Benlloch, I. (Coords.) (2004). Psicología y género. Madrid: Pearson Educación.

Bascón, M.; Arias, S. \& De la Mata, M. (2013). Contenidos y modos conversacionales en adolescentes: debatiendo sobre conflictos grupales y violencia de pareja. Infancia $y$ Aprendizaje: Journal for the Study of Education and Development, 36(4), 489-500. doi: http://dx.doi.org/10.1174/021037013808200302

Bergesio, L. \& Golovanevsky, L. (2008). Mapa de la informalidad en Argentina (2003-2006): un 
ejercicio preliminar a partir del análisis multivariado de datos. En Anales XLIII Reunión Anual de AAEP y IX Reunión Anual sobre Pobreza y Distribución del Ingreso de la Network on Inequality and Poverty. Córdoba: Universidad Nacional de Córdoba. Recuperado de: http://www.aaep.org.ar/anales/works/works2008/ bergesio.pdf

Bergesio, L. \& Golovanevsky, L. (2013). Informalidad en Argentina: una aproximación desde el análisis multivariado de datos en los primeros años de la recuperación económica (2003-2006).Trabajo y Sociedad, 21(17), 437$457 . \quad$ Recuperado de: http://www.unse.edu.ar/trabajoysociedad/21\%20 BERGESIO\%20Y\%20GOLOVANEVSKY\%20t rabajo\%20informal.pdf

Berk, L.E. (2009). Desarrollo del niño y del adolescente. Madrid: Pearson Educación.

Bogardus, E. (1965). The development of social thought. Nueva York: Davis McKay Company.

Bonino, L. (2005). Las microviolencias y sus efectos: claves para su detección. En C. RuizJarabo \& P. Blanco (Coords.), La violencia contra las mujeres: prevención y detección (pp. 83-102). Madrid: Díaz de Santos.

Bosch, E.; Ferrer, V.A. \& Alzadora, A. (2006). El laberinto patriarcal. Reflexiones teóricoprácticas sobre la violencia contra las mujeres. Barcelona: Anthropos.

Bueno, M.R. \& Garrido, M.A. (2012). Relaciones interpersonales en la educación. Madrid: Pirámide.

Castells, M. \& Subirats, M. (2007). Mujeres y hombres: ¿un amor imposible? Madrid: Alianza Editorial.

Ceballos, E.M. (2014). Coeducación en la familia: una cuestión pendiente para la mejora de la calidad de vida de las mujeres. Revista electrónica interuniversitaria de formación del profesorado,17(1), 1-14. doi: http://dx.doi.org/10.6018/reifop.17.1.198811

Chaves, A.R. (2012). Masculinidad y feminidad: ¿de qué estamos hablando? Revista Electrónica Educare, 16(número especial), 5-13. Recuperado de:

http://www.revistas.una.ac.cr/index.php/EDUCA RE/article/download/4746/4569

Colás, P. (2007). La construcción de la identidad de género: Enfoques teóricos para fundamentar la investigación e intervención educativa. Revista de Investigación Educativa, 25(1), 151-166. Recuperado de: http://revistas.um.es/rie/article/view/96661/92871

Collins, R. (1974). Three faces of cruelty: towards a comparative sociology of violence. Theory and Society, 1(4), 415-440. doi: http://dx.doi.org/10.1007/BF00160802

Consejería de Educación de la Junta de Andalucía (2006). Regulación de las actuaciones y medidas establecidas en el I Plan de Igualdad entre Hombres y Mujeres en Educación. Orden de 15 de mayo de 2006 del Consejo de Gobierno. BOJA número 99 de 25 de mayo de 2006 (pp. 12-14). Recuperado de: http://www.juntadeandalucia.es/boja/2006/99/4

Cornejo, J.M. (1988). Técnicas de investigación social: el análisis de correspondencias. Teoría y práctica. Barcelona: PPU.

Cornejo, J.M. (2003a). Análisis sociométricos. Guía de trabajo. Documento del Laboratorio de Psicología Social, Universidad de Barcelona. Recuperado de: http://www.ub.es/dppss/lps/docu/asoc.pdf

Cornejo, J.M. (2003b). Técnicas de análisis grupal. Guía de trabajo. Documento del Laboratorio de Psicología Social, Universidad de Barcelona. Recuperado de: http://www.ub.es/dppss/lps/docu/tag.pdf

Cornejo, J.M. (2006). El análisis de las interacciones grupales: las aplicaciones SOCIOS. Anuario de Psicología, 37(3), 277-297. Recuperado de: http://www.raco.cat/index.php/AnuarioPsicologia /article/view/61842/82854

Crawford, M. (2006). Transformations. Women, Gender and Psychology. Boston: McGraw-Hill.

Crawford, M. \& Chaffin, R. (1997). The Meanings of Difference: Cognition in Social and Cultural Context. En P. Caplan, M. Crawford, J. Shibley Hyde \& J. Richardson (Eds.), Gender Differences in Human Cognition (pp. 81-130). Oxford: Oxford University Press.

De la Peña, E.M.; Ramos, E.; Luzón, J.M. \& Recio, P. (2011). Andalucía Detecta - Andalucía Previene: sexismo y violencia de género en la juventud. Sevilla: Instituto Andaluz de la Mujer. Recuperado de: http://www.juntadeandalucia.es/institutodelamuje 
García-Pérez, Rafael; Ruiz-Pinto, Estrella \& Rebollo-Catalán, Ángekes (2016). Preferencias relacionales de género en el contexto escolar: Una nueva medida para el diagnóstico de relaciones de género en educación. RELIEVE, 22 (1), art. 3. DOI: http://dx.doi.org/10.7203/relieve22.1.6877

r/index.php/component/remository/funcstartdown/1670/?Itemid=71

Díaz-Aguado, M.J. \& Martín-Seoane, G. (2011). Convivencia y aprendizaje escolar en la adolescencia desde una perspectiva de género. Psicothema, 23(2), 252-259. Recuperado de: http://www.psicothema.com/pdf/3879.pdf

Díaz-Martínez, C. \& Dema, S. (Coords.) (2013). Sociología y género. Madrid: Tecnos.

Fernández-Sánchez, J. (2011). Un siglo de investigaciones sobre masculinidad y feminidad: una revisión crítica. Psicothema, 23(2), 167-172. Recuperado http://www.psicothema.com/pdf/3866.pdf

Ferrer, V.A. \& Bosch, E. (2013). Del amor romántico a la violencia de género: para una coeducación emocional en la agenda educativa. Profesorado. Revista de currículum y formación del profesorado, 17(1), 105-122. Recuperado de: http://www.ugr.es/ recfpro/rev171ART7.pdf

Ferrer, V.A.; Bosch, E.; Navarro, C.; Ramis, M.C. \& García, M.E. (2008). Los micromachismos o microviolencias en la relación de pareja: una aproximación empírica. Anales de Psicología, 24(2), 341-352. Recuperado de: http://www.um.es/analesps/v24/v24_2/1824_2.pdf

Garaigordobil, M. (2012). Resolución de conflictos cooperativa durante la adolescencia: relaciones con variables cognitivo-conductuales y predictores. Infancia y Aprendizaje, 35(2), 151165. doi: http://dx.doi.org/10.1174/021037012800217998

Garaigordobil, M. \& Aliri, J. (2013). Relaciones del sexismo con justificación de la violencia, y con otras formas de prejuicio como la dominancia social y el autoritarismo. Estudios de Psicología,34(2), 127-139. doi: http://dx.doi.org/10.1174/021093913806751384

García-Pérez, R.; Rebollo-Catalán, A.; BuzónGarcía, O.; González-Piñal, R.; BarragánSánchez, R. \& Ruiz-Pinto, E. (2010). Actitudes del alumnado hacia la igualdad de género. Revista de Investigación Educativa, 28(1), 217$232 . \quad$ Recuperado de: http://revistas.um.es/rie/article/view/98951

García-Santesmases, A.; Herrero, C.; Olaso, J. \& Martínez-Ten, L. (2012). La construcción de la identidad de género desde una perspectiva intercultural: propuestas didácticas de intervención educativa. Madrid: Fete Enseñanza. Recuperado de: http://www.educandoenigualdad.com/wpcontent/uploads/2015/03/IDENTIDAD_GENER O_PERSPECTIVA_INTERCULTURAL.pdf

García-Tornel, S.; Miret, P.; Cabré, A.; Flaquer, L.; Berg-Kelly, K.; Roca, G.; Elzo, J. \& Lailla,, J.M. (Coords.) (2011). El adolescente y su entorno en el siglo XXI: instantánea de una década. Esplugues de Llobregat: Hospital Sant Joan de Déu. Recuperado de: http://faros.hsjdbcn.org/sites/default/files/faros_ 5 cast.pdf

Guasch, O. (2007). La crisis de la heterosexualidad. Barcelona: Laertes.

Herrera, M.C.; Expósito, F. \& Moya, M. (2012). Negative reactions of men to the loss of power in gender relations: Lilith vs. Eve. The European Journal of Psychology Applied to Legal Context, 4(1), 17-42. Recuperado de: http://www.webs.uvigo.es/sepjf/index.php?option =com docman\&task=doc download\&gid=42\&It emid=110\&lang $=$ es

Jensen-Arnett, J. (2008). Adolescencia y adultez emergente: un enfoque cultural. Naucalpan de Juárez: Pearson Educación.

Jiménez-Cortés, R.; Rebollo-Catalán, A.; GarcíaPérez, R. \& Buzón-García, O. (2015). Motivos de uso de las redes sociales virtuales: análisis de perfiles de mujeres rurales. RELIEVE, 21(1). doi: http://dx.doi.org/10.7203/relieve.21.1.5153

Joaristi, L. \& Lizasoain, L. (1999). Análisis de correspondencias. Madrid: La Muralla.

Lagarde, M. (1996). Género y feminismo. Desarrollo humano y democracia. Madrid: Horas y Horas.

Ley Orgánica 3/2007, de 22 de marzo (texto consolidado a 27 de julio de 2013), para la Igualdad Efectiva de Mujeres y Hombres. BOE número 71 de 23 de marzo de 2007 (pp. 1261112645). Recuperado de: http://www.boe.es/buscar/act.php?id=BOE-A2007-6115

Letelier, M.J.; Aller, M.B.; Henao, D.; SánchezPérez, I.; Vargas, I.; Coderch de Lassaletta, J.; Llopart, J.R.; Ferran, M.; Colomés, L. \& Vázquez, M.L. (2010). Diseño y validación de un cuestionario para medir la continuidad asistencia entre niveles desde la perspectiva del usuario: CCAENA. Gaceta Sanitaria, 24(4), 339-346. 
doi:

http://dx.doi.org/10.1016/j.gaceta.2010.03.010

Lomas, C. (2007). ¿La escuela es un infierno?: violencia escolar y construcción cultural de la masculinidad. Revista de Educación, 342, 83$101 . \quad$ Recuperado de: http://www.revistaeducacion.mec.es/re342/re342 _05.pdf

Lorente, M. (2007). Violencia de género, educación y socialización: acciones y reacciones. Revista de Educación, 342(1), 19-35. Recuperado de:

http://www.revistaeducacion.mec.es/re342/re342 _02.pdf

Lorente, M. (2009). Los nuevos hombres nuevos: cómo adaptarse a los tiempos de igualdad. Barcelona: Destino.

Martínez-Benlloch, I. \& Bonilla, A. (2000). Sistema sexo/género, identidades y construcción de la subjetividad. Valencia: Publicaciones de la Universidad de Valencia.

Martínez-Sánchez, I.; Navarro, R. \& Yubero, S. (2009). Estereotipos de género entre los adolescentes españoles: imagen prototípica de hombres y mujeres e imagen de uno mismo. Informació psicológica, 95, 77-86. Recuperado de:

https://ruidera.uclm.es/xmlui/bitstream/handle/10 $\underline{578 / 1957 / f i \quad 1331640284-}$

10.\%20informacio\%20psicologica.pdf?sequence $=1$

Meseguer, D. \& Villar, A. (2011). Género y educación. En J. Beltrán \& F.J. Hernández (Coords.), Sociología de la educación (pp. 155184). Madrid: McGraw-Hill.

Moreno, J.L. (1934). Who Shall Survive? A new approach to the problem of human relationships. Washington, DC: Nervous and Mental Dis.Pub.

Morgade, G. (2009). Aprender a ser mujer, aprender a ser varón: relaciones de género y educación. Esbozo de un programa de acción. Madrid: Cep.

Muñoz-Tinoco, M.V.; Jiménez-Lagares, I. \& Moreno, M.C. (2008). Reputación conductual y género en la adolescencia. Anales de psicología, 24(2), 334-340. Recuperado de: http://revistas.um.es/analesps/article/view/42891/ $\underline{41211}$

Murillo, F.J. \& Hernández-Castilla, R. (2015). Liderazgo para el aprendizaje: ¿qué tareas de los directores y directoras escolares son las que más inciden en el aprendizaje de los estudiantes?RELIEVE, 21(1). doi: http://dx.doi.org/10.7203/relieve.21.1.5015

Piedra, J. (Coord.) (2013). Géneros, masculinidades y diversidad: educación física, deporte e identidades masculinas. Barcelona: Octaedro.

Rodríguez-Mosquera, P.M. (2011). Códigos de honor masculinos y femeninos. Revista de Psicología Social, 26(1), 63-72. doi: http://dx.doi.org/10.1174/021347411794078499

Ruiz-Pinto, E.; García-Pérez, R. \& RebolloCatalán, A. (2013). Relaciones de género de adolescentes en contextos educativos: análisis de redes sociales con perspectiva de género. Profesorado. Revista de currículum y formación del profesorado, 17(1), 123-140. Recuperado de: http://www.ugr.es/local/recfpro/rev171ART8.pdf

Sánchez-Carrión, J.J. (1989). Análisis de tablas de contingencia: el uso de los porcentajes en las ciencias sociales. Madrid: Centro de Investigaciones Sociológicas y Siglo XXI de España.

Simón, M.E. (2008). Hijas de la igualdad, herederas de injusticias. Madrid: Narcea.

Simón, M.E. (2010). La igualdad también se aprende: cuestión de coeducación. Madrid: Narcea.

Soler, S. (2009). Los procesos de reproducción, resistencia y cambio de las relaciones tradicionales de género en Educación Física: el caso del fútbol. Cultura y Educación, 21(1), 31$42 . \quad$ doi: http://dx.doi.org/10.1174/113564009787531253

Subirats, M. (2009). La escuela mixta: ¿ ¿garantía de coeducación? Participación Educativa, 11, 94$97 . \quad$ Recuperado de: http://www.mecd.gob.es/revista-cee/pdf/n11subirats-martori.pdf

Subirats, M. (2010). ¿Coeducación o escuela segregada?: un viejo y persistente debate. Revista de la Asociación de Sociología de la Educación, 3(1), 143-158. Recuperado de: http://www.ase.es/rase/index.php/RASE/article/vi ew/133/128

Subirats, M. (2011). Igualdad de sexos, desigualdad de géneros. Tiempo de paz, 100, 183-187. 
Subirats, M. \& Tomé, A. (2007). Balones fuera; reconstruir los espacios desde la coeducación. Barcelona: Octaedro.

Tomé, A. \& Tonucci, F. (2013). Con ojos de niña. Barcelona: Graó.

West, C. \& Zimmerman, D.H. (1987). Doing Gender. Gender and Society, 1(2), 125-151.

Zambrini, L. (2010). Modos de vestir e identidades de género: reflexiones sobre las marcas culturales sobre el cuerpo. Nomadías: revista del centro de estudios de género y cultura de América Latina, 11, 130-149. doi: http://dx.doi.org/10.5354/0719$\underline{0905.2010 .15158}$

Zambrini, L. (2013). El género como metáfora: narrativas sobre travestis en prensa digital argentina (2004-2009). Sociedad y Economía, 24, 143-158. Recuperado de: http://sociedadyeconomia.univalle.edu.co/index.p hp/sye/article/view/2301/2151

\section{Agradecimientos}

Este trabajo se ha realizado gracias al proyecto de investigación de excelencia TEÓN XXI: Creación de recursos online para el conocimiento y difusión de la cultura de género en la escuela(número P06-HUM-01408, BOJA n ${ }^{\circ} 71$ de 11 de abril de 2007), subvencionado por la Consejería de Innovación, Ciencia y Empresa de la Junta de Andalucía. Queremos también agradecer al profesorado y alumnado de los centros públicos de Educación Secundaria de Andalucía su participación en el presente estudio así como al personal técnico experto en igualdad $\mathrm{y}$ coeducación de la Junta de Andalucía por su asesoramiento

\section{Autores / Authors}

To know more / Saber más

García-Pérez, Rafael (rafaelgarcia@us.es).

Profesor Titular en la Universidad de Sevilla (España). Su línea de investigación se centra en Técnicas e Instrumentos de Diagnóstico sobre Género, Tecnología y Educación. Su dirección postal es Dpto. Métodos de Investigación y Diagnóstico en Educación. Facultad de Ciencias de la Educación Universidad de Sevilla, C/ Pirotecnia, s/n, CP 41013, Sevilla (España)

ORCID

$\underline{0000-0002-6282-3016}$

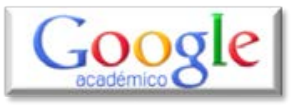

ResearchGate

Ruiz-Pinto, Estrella (epinto@us.es).

Licenciada en Pedagogía y Master en Estudios de Género en la especialidad de Intervención en los Procesos Educativos. Su línea de investigación relaciona la perspectiva de género con el análisis de redes sociales (ARS). Dirección: Dpto. MIDE. Facultad CC.EE. Universidad de Sevilla, C/ Pirotecnia, s/n, CP. 41013, Sevilla (España)

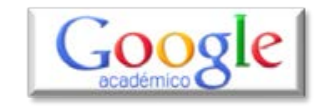

Rebollo-Catalán, Ma Ángeles (rebollo@us.es).

Profesora Titular de Universidad en la Universidad de Sevilla (España). Su principal línea de investigación se centra en Género, Tecnología y Educación. Su dirección postal es Dpto. Métodos de Investigación y Diagnóstico en Educación. Facultad de Ciencias de la Educación Universidad de Sevilla, C/ Pirotecnia, s/n, CP. 41013, Sevilla (España)

$\underline{0000-0003-3523-0714}$

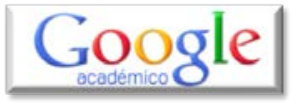

Researchtate 


\section{RELIEVE}

\section{Revista ELectrónica de Investigación y EValuación Educativa E-Journal of Educational Research, Assessment and Evaluation}

[ISSN: 1134-4032]

(C) Copyright, RELIEVE. Reproduction and distribution of this articles it is authorized if the content is no modified and their origin is indicated (RELIEVE Journal, volume, number and electronic address of the document).

(C) Copyright, RELIEVE. Se autoriza la reproducción y distribución de este artículo siempre que no se modifique el contenido y se indique su origen (RELIEVE, volumen, número y dirección electrónica del documento). 